\title{
Shear Plate Interferometer for Complex Angular Momentum Field Generation
}

\author{
William R. Kerridge-Johns*, Jean-Benoît Jaillot, Michael J. Damzen \\ Photonics Group, The Blackett Laboratory, Imperial College London, SW7 2AZ, UK \\ *wrk10@ic.ac.uk
}

\begin{abstract}
We show that a shear plate interferometer can generate high purity complex angular momentum states and is simple, cheap, compact, and could operate in wavelength regions where optics for conventional techniques are not available. (C) 2020 The Authors
\end{abstract}

\section{Introduction}

Vortex beams have been a topic of great interest to the scientific community since the identification of their orbital angular momentum. To match this application interest, there has been a wide variety of generation techniques developed. Some convert the entirety of the input beam into a vortex in one step using a single or group of optical elements, such as astigmatic lensing mode converters, spiral phase plates, q-plates, and those exploiting birefringence properties. Another principle is to convert a portion of the incoming beam into the desired vortex mode using a specialised diffraction grating, which can either be fixed or is much more commonly a computer controllable spatial light modulator that can rapidly generate custom and adaptable intensity and phase patterns.

A more recent development has been to use interferometry to convert one laser mode into another. It has been shown that the superposition of two Gaussian beams can yield a vortex beam [1], and we have recently shown that more complex vortex mode superpositions can be obtained through transforming vortex modes themselves [2]. These approaches have been experimentally implemented using Mach-Zehnder and Sagnac interferometers, which have the advantage of being highly customisable but introduce the issues of requiring alignment of multiple optical elements and the physical volume they must occupy.

In this work we investigate the interferometric mode conversion technique using a Shear Plate Interferometer (SPI), which is simply an optical wedge typically with a thickness in the order of millimeter and a wedge angle in the order of milliradians. SPIs are widely commercially available in a range of materials, wedge angles, and thicknesses, and they can be used uncoated giving them a high surface optical damage threshold equal to the base material. This device will reflect a beam of increased or decreased vorticity a specified condition is met and is highly stable due to the monolithic nature of the interferometer. This technique could also be applied in any wavelength regime, for example in the far infrared where spatial light modulators are not available.

\section{Shear Plate Interferometer}

The operation of the SPI is illustrated in Fig. 1(a). A beam is incident on the plate and a fraction of this input is reflected in a transformed state, with the remaining power transmitted approximately unaltered. The spatial

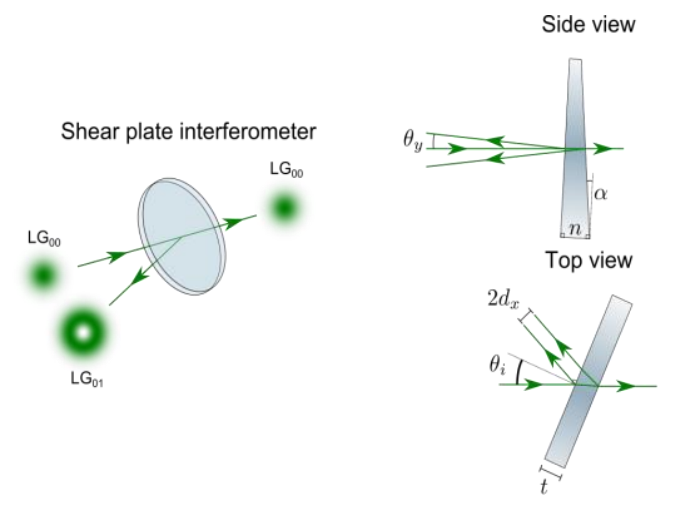

(a)

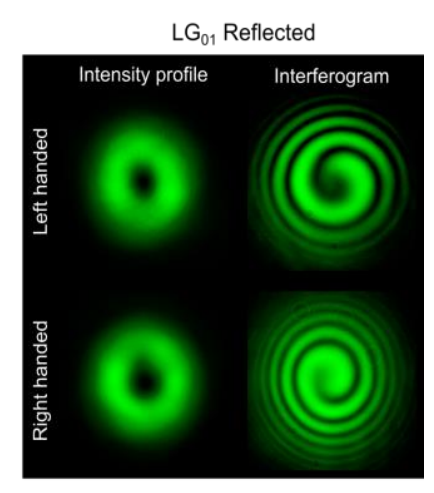

(c)

Fig. 1. The shear plate interferometer (SPI) functionality and results: (a) $\mathrm{LG}_{00}$ input to the SPI is converted into an $\mathrm{LG}_{01}$ reflection, (b) the side and top views of the SPI showing the angular offset from the wedged faces (top image) and the shear displacement from the angle of incidence (bottom image), (c) experimental $\mathrm{LG}_{01}$ generation results with $\mathrm{LG}_{00}$ input showing left and right handed phase interferograms. 
transform is the result of the interferometric combination of the reflected beams from the front and rear surfaces of the SPI, which is designed to be a combination of a shear and angular offset in orthogonal axes as illustrated in Fig. 1 (b). The angular offset $\theta_{y}$ comes from the wedge angle on the plate $\alpha$, and the shear offset $d_{x}$ is from an angle of incidence $\theta_{i}$ onto the plate of nominal thickness $t$. When these parameters satisfy

$$
\frac{t \theta_{i}}{n w_{0}}=\frac{\pi w_{0} n \alpha}{\lambda}=\epsilon
$$

where $w_{0}$ is the beam waist radius, $n$ is the plate refractive index, $\lambda$ is the wavelength, and $\epsilon \ll 1$ determines the scale of the offsets, then an input $\mathrm{LG}_{00}$ beam will be transformed into an $\mathrm{LG}_{01}$ mode.

The transformation was verified experimentally using a commercially available SPI (Thorlabs SI100P), which had a nominal thickness of $2.6 \mathrm{~mm}$ and a wedge angle of $40 \mathrm{arcsec}(0.19 \mathrm{mrad})$. The Gaussian source was a $543 \mathrm{~nm}$ green Helium-Neon laser, which had a waist of radius $118 \mu \mathrm{m}$ at the SPI. The resulting transformed modes are shown in Fig. 1(c). The $\mathrm{LG}_{01}$ modal purity was excellent and measured to be greater than $95 \%$ using a modal decomposition technique based on phase retrieval. The interferograms were obtained with a Mach-Zehnder interferometer to visually show the spiral phase profile, and the vortex handedness could be reversed simply by rotating the plate to reverse the angle of incidence direction.

\section{Complex Angular Momentum Field Generation}

The angular momentum transformation concept can be extended to create complex angular momentum states. The condition of Eq. (1) causes higher azimuthal order $\mathrm{LG}_{01}$ modes to be transformed into discrete superpositions of other LG modes, which can be selected to either increase or decrease the vorticity of the input - the total number of $2 \pi$ phase changes around the beam perimeter. An example set of these transformations is shown in Fig. 2, which in initial investigations were obtained with a Sagnac interferometer operating identically to a SPI. For example, the $\mathrm{LG}_{01}$ mode can be transformed into a superposition of the $\mathrm{LG}_{00}$ and $\mathrm{LG}_{02}$ modes.

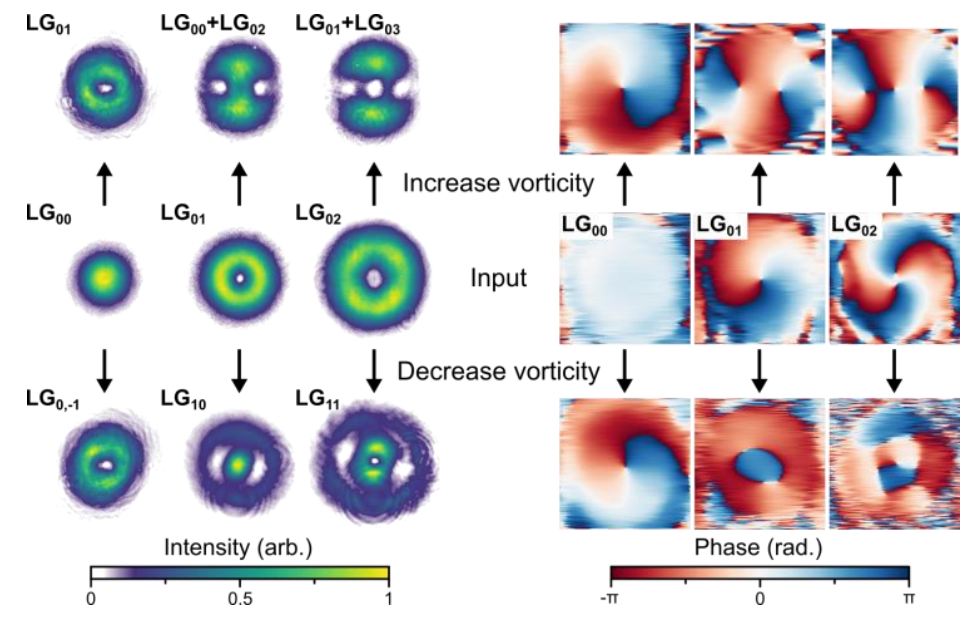

Fig. 2. The complex angular momentum states that can be generated with the SPI type of transformation.

Left: Input Laguerre-Gaussian modes have their vorticity increased into a superposition state or decreased into a higher order radial LG mode. Right: The obtained phase of the beams verifying the phase singularities.

\section{Conclusion}

In this work we show that the SPI is a readily available, compact single optical component, and simple device to operate, yet provides a platform to generate and investigate a rich set of angular momentum states. We anticipate that this device may be of interest in education due to its simplicity of operation, in addition to scientific and commercial applications that require a compact, robust, and high-quality vortex beam. The SPI in this work operated with Fresnel surface reflection, therefore complex angular momentum studies could be performed in wavelength regions where conventional optics are not available, for example in the far infrared.

[1] P. Vaity et. al., "Formation of optical vortices through superposition of two Gaussian beams," Appl. Opt. 52, $6652-6656$ (2013).

[2] M. J. Damzen et. al. "Vortex mode transformation interferometry," J. Opt. 22, 015604 (2020). 